\title{
NeuroTec Sitem-Insel Bern: Closing the Last Mile in Neurology
}

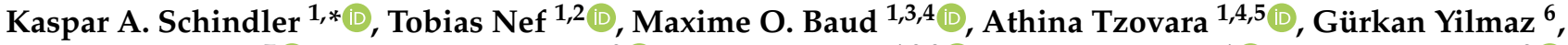

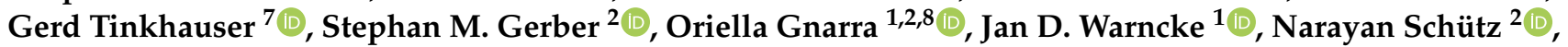

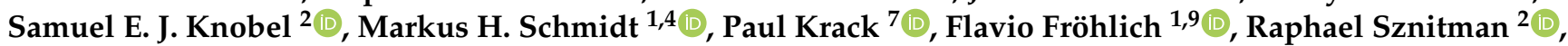 \\ Simon Rothen ${ }^{10}$ and Claudio L. A. Bassetti ${ }^{1,11} \mathbb{D}$
}

check for

updates

Citation: Schindler, K.A.; Nef, T.; Baud, M.O.; Tzovara, A.; Yilmaz, G.;

Tinkhauser, G.; Gerber, S.M.;

Gnarra, O.; Warncke, J.D.; Schuetz, N. et al. NeuroTec Sitem-Insel Bern: Closing the Last Mile in Neurology. Clin. Transl. Neurosci. 2021, 5, 13. https://doi.org/10.3390/ctn5020013

Academic Editor: Dominik Straumann

Received: 9 June 2021

Accepted: 16 July 2021

Published: 22 July 2021

Publisher's Note: MDPI stays neutra with regard to jurisdictional claims in published maps and institutional affiliations.

Copyright: (C) 2021 by the authors Licensee MDPI, Basel, Switzerland. This article is an open access article distributed under the terms and conditions of the Creative Commons Attribution (CC BY) license (https:// creativecommons.org/licenses/by/ $4.0 /)$.
1 Sleep-Wake-Epilepsy Center I NeuroTec, Department of Neurology, Inselspital Bern, University Hospital, University of Bern, 3010 Bern, Switzerland; tobias.nef@artorg.unibe.ch (T.N.); maxime.baud@insel.ch (M.O.B.); athina.tzovara@inf.unibe.ch (A.T.); oriella.gnarra@insel.ch (O.G.); jan.warncke@insel.ch (J.D.W.); markus.schmidt@insel.ch (M.H.S.); flavio_frohlich@med.unc.edu (F.F.); claudio.bassetti@insel.ch (C.L.A.B.)

2 ARTORG Center for Biomedical Engineering Research, University of Bern, 3008 Bern, Switzerland; stephan.gerber@artorg.unibe.ch (S.M.G.); narayan.schuetz@artorg.unibe.ch (N.S.); samuel.knobel@artorg.unibe.ch (S.E.J.K.); raphael.sznitman@artorg.unibe.ch (R.S.)

3 Wyss Center for Bio- and Neuro-Engineering, 1202 Geneva, Switzerland

4 Center for Experimental Neurology, Department of Neurology, Inselspital Bern, University Hospital, University of Bern, 3010 Bern, Switzerland

5 Institute for Computer Science, University of Bern, 3012 Bern, Switzerland

6 Swiss Center for Electronics and Microtechnology (CSEM), 2002 Neuchâtel, Switzerland; guerkan.yilmaz@csem.ch

7 Center for Parkinson's Disease and Movement Disorders, Department of Neurology, Inselspital Bern, University Hospital, University of Bern, 3010 Bern, Switzerland; gerd.tinkhauser@insel.ch (G.T.); paul.krack@insel.ch (P.K.)

8 Sensory-Motor System Lab, IRIS, ETH Zurich, 8092 Zurich, Switzerland

9 University of North Carolina at Chapel Hill, Chapel Hill, NC 27599-7250, USA

10 Swiss Institute for Translational and Entrepreneurial Medicine, Sitem-Insel, 3010 Bern, Switzerland; simon.rothen@sitem-insel.ch

11 Neurology Department, Sechenov University Moscow, 119435 Moscow, Russia

* Correspondence: kaspar.schindler@insel.ch

Abstract: Neurology is focused on a model where patients receive their care through repeated visits to clinics and doctor's offices. Diagnostic tests often require expensive and specialized equipment that are only available in clinics. However, this current model has significant drawbacks. First, diagnostic tests, such as daytime EEG and sleep studies, occur under artificial conditions in the clinic, which may mask or wrongly emphasize clinically important features. Second, early detection and high-quality management of chronic neurological disorders require repeat measurements to accurately capture the dynamics of the disease process, which is impractical to execute in the clinic for economical and logistical reasons. Third, clinic visits remain inaccessible to many patients due to geographical and economical circumstances. Fourth, global disruptions to daily life, such as the one caused by COVID-19, can seriously harm patients if access to in-person clinical visits for diagnostic and treatment purposes is throttled. Thus, translating diagnostic and treatment procedures to patients' homes will convey multiple substantial benefits and has the potential to substantially improve clinical outcomes while reducing cost. NeuroTec was founded to accelerate the re-imagining of neurology and to promote the convergence of technological, scientific, medical and societal processes. The goal is to identify and validate new digital biomarkers that can close the last mile in neurology by enabling the translation of personalized diagnostics and therapeutic interventions from the clinic to the patient's home.

Keywords: dynamical disease; digital biomarkers; home diagnostics; ultra-long-term EEG; machine learning; public-private partnerships 


\section{Introduction}

Most neurological and neuro-psychiatric disorders, such as epilepsy, Parkinson's disease, depression, migraine, multiple sclerosis, Alzheimer's disease and also the sequelae of stroke, are not transient but chronic [1-4]. Importantly, their time courses typically are not linear, but are rather characterized by fluctuations, modulations by sleep and multidien periodicities, often evolving in highly patient-specific ways [5-9]. These individual dynamics may inform personalized diagnostics and therapies on an unprecedented scale. However, in today's prevailing health care system with hospital- and appointmentbased neurology, it is most often not possible to accurately assess the structure of the time course of chronic neurological disorders, i.e., their dynamics are under-sampled. What is, therefore, urgently needed are novel technologies that allow for longitudinal monitoring of neurological disorders in real-world environments of patients. NeuroTec is a new research hub, located at the Swiss Institute for Translational and Entrepreneurial Medicine (sitem) directly on the campus of the Inselspital, the University Hospital of Bern (Figure 1). NeuroTec comprises a testing platform with an instrumented apartment, and sleep, EEG and circadian labs, designed to accelerate the translation of diagnostics and therapies from the clinic to the patient's home. NeuroTec is run by the Department of Neurology of the Inselspital in close collaboration with the ARTORG Center for Biomedical Engineering of the University of Bern, with project-specific partners from academia, non-profit research institutions and industrial partners in public-private partnerships. The first generation of projects, which we here set out to describe, span both recording and stimulation, invasive and non-invasive methods, and thus provide a firm yet innovative platform to transform neurology through synergy and collaboration. The main goal of NeuroTec is to develop, test and deliver such technologies and to thus pave the way for patient-centered neurology that aims to diagnose and treat patients in their homes.

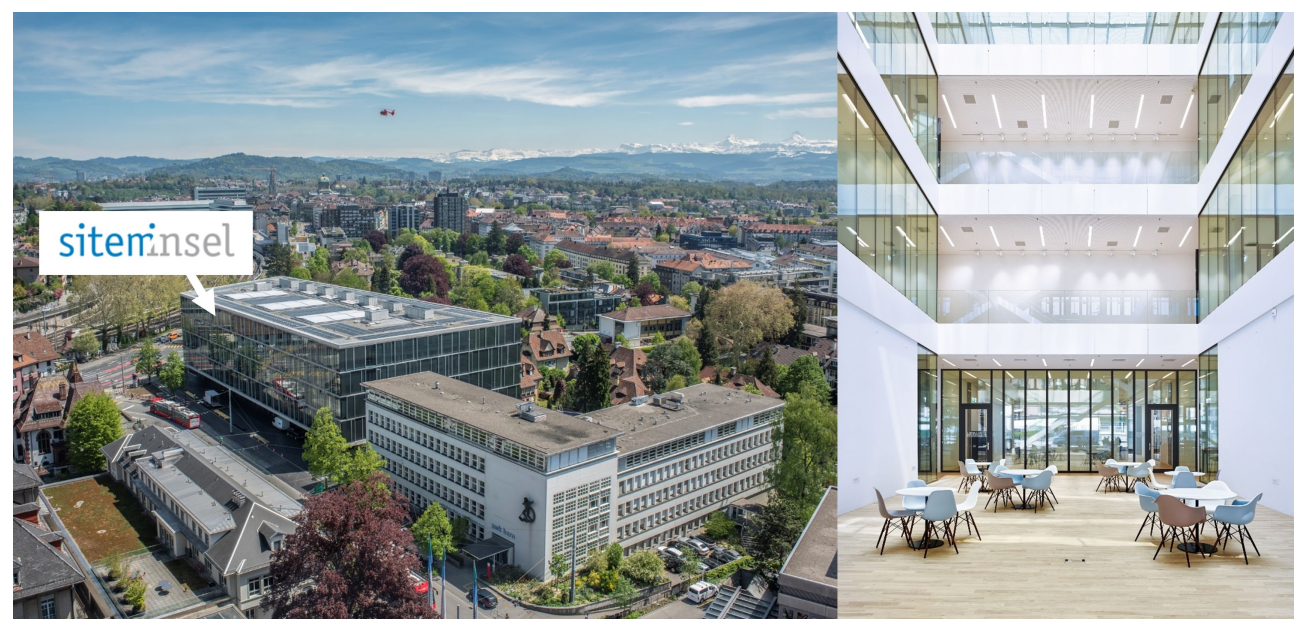

Figure 1. NeuroTec is located at the new Swiss Institute for Translational and Entrepreneurial Medicine (sitem) on the campus of Inselspital, the University Hospital of Bern.

\section{Motion and Emotion-Digital Biomarkers for Closed-Loop Deep Brain Stimulation}

One core component of NeuroTec is its "Loft" -an instrumented apartment (Figure 2), which will be used for studies investigating the behavioral aspects of neurological and neuro-psychiatric disorders. This unique facility enables the investigation of patients in conditions that closely mimic their natural living situation. In one project, we use the NeuroTec Loft to objectively investigate motor and non-motor symptoms of Parkinson's disease (PD) and use this information to individually optimize deep brain stimulation (DBS) parameters. PD is the second most common neurodegenerative disorder characterized by a loss of dopaminergic cells in the brain, causing motor (e.g., bradykinesia, and tremor) but also non-motor symptoms (e.g., sleep disorders and neuropsychiatric disturbances). In the early phase of the disease, typically during the first $3-5$ years, pharmacological treat- 
ments, including the use of levodopa (prodrug of dopamine) and other dopaminergic therapies are sufficient to control symptoms and to restore a good quality of life. With the progression of the disease, however, the brain of people with Parkinson's loses its ability to compensate for the temporal intermittent oral intake of dopaminergic medication, and, as a result, varying drug levels in the blood lead to troublesome fluctuations of both motor and non-motor symptoms throughout the day. For patients in this advanced stage of the disease, DBS represents a proven and established treatment option to better (but not entirely) control these symptom fluctuations [2]. Nowadays, DBS systems in clinical use for movement disorders exclusively operate in an open-loop mode; thus, stimulation can only be delivered continuously without automatized adjustments to the clinical state, thereby limiting a stable symptom control [2]. Moreover, current DBS systems are limited by manual and time-consuming iterative adjustments of the stimulation parameters, which establish a chronic stimulation setting [10]. Closed-loop DBS represents a new technology on the horizon that is likely to address the fluctuating symptom states more specifically by titrating the delivery of stimulation according to symptom biomarkers extracted from brain or other signal sources in real time [11]. Major progress has been made in linking patterns in brain oscillations recorded from the DBS target structures to symptoms in PD [12,13]; the first pilot trials using brain signal driven closed-loop DBS showed promising results [14]. These developments were important impulses for the device manufacturers, as new neurostimulators with brain sensing capabilities have now been released for commercial use and for further development of closed-loop DBS algorithms. As an additional goal, we aim to complement brain signal recordings with sensor-based recordings during the patient's daily life. Sensor-based quantification of PD motor symptoms is a well-studied problem (see Ossig C. et al., 2016 [15] and Rovini et al., 2017 [16] for reviews), but as of yet there are no established methods for unobtrusive sensor-based monitoring of non-motor symptoms. In this project, we aim to develop and evaluate the feasibility of a new closed-loop DBS control system that uses both internal and external signals to minimize PD symptoms and maximize patients' quality of life, thus fostering independent living. To achieve this goal, we use sensor fusion and machine learning algorithms to quantify emotions (e.g., happiness, anger, apathy, depression), motor symptoms (e.g., tremor, and bradykinesia) and cognitive abilities (e.g., memory, processing speed). These measures, together with the internally recorded neural oscillations, serve as inputs to a heuristic controller implemented in the DBS system. The control objective is to minimize symptoms in the aforementioned domains. We propose to analyze continuous multi-modal recordings of speech (with an array of microphones), activities of daily living and sleep (with ambient sensors), body movements and cardiac activity (with a wearable sensor at the wrist as well as ambient sensors integrated in the bed) and discrete measurements of cognitive performance (with a tablet- or smartphone-based computer game). Fluctuations of non-motor symptoms, including emotional disturbances, neuropsychiatric and behavioral symptoms, and cognitive performance are paramount for the quality of life but are, at the same time, often under-diagnosed in PD. The idea of using emotion detection in spontaneous speech and leveraging this new item with context information from other sensor modalities (e.g., ambient and wearable sensors) opens a novel approach to better understanding and treating the neuropsychiatric symptoms in PD. In the first step, we plan to recruit 20 PD patients to conduct a thorough behavioral monitoring and profiling study. To this end, the behavioral measurements will take place in the patient's home (total duration of 12 weeks) and in the sensor-equipped apartment (total duration of 4 days) at NeuroTec (Figure 2). In addition to the aforementioned sensors, which will be used in the patient's home (see also Schütz et al., 2019 [17]), there will be more sophisticated sensors in the NeuroTec Loft, providing fine-grained information about the patient's activities. We hypothesize that the combination of fine-graded, but relatively short (e.g., 4 days), measurements in the NeuroTec Loft with the longer duration (e.g., 12 weeks), but less sophisticated measurements in the patient's home will help us to quantify both the temporal fluctuations and the characteristics of the patients' individual motor and 
non-motor symptoms. As a second step, this same behavioral profiling approach will be used for patients with DBS to allow for more fine-tuned and individualized calibration of DBS stimulation parameters to improve the quality of life. Importantly, similar projects could be envisioned for studying the behavioral correlates of other neurological and psychiatric "loop disorders" [13], such as dystonia, Tourette's syndrome, depression or epilepsy. This approach is uniquely enabled by the clinical and engineering expertise that are blended through the structure of the NeuroTec, which fosters such collaborations and provides the required labs.
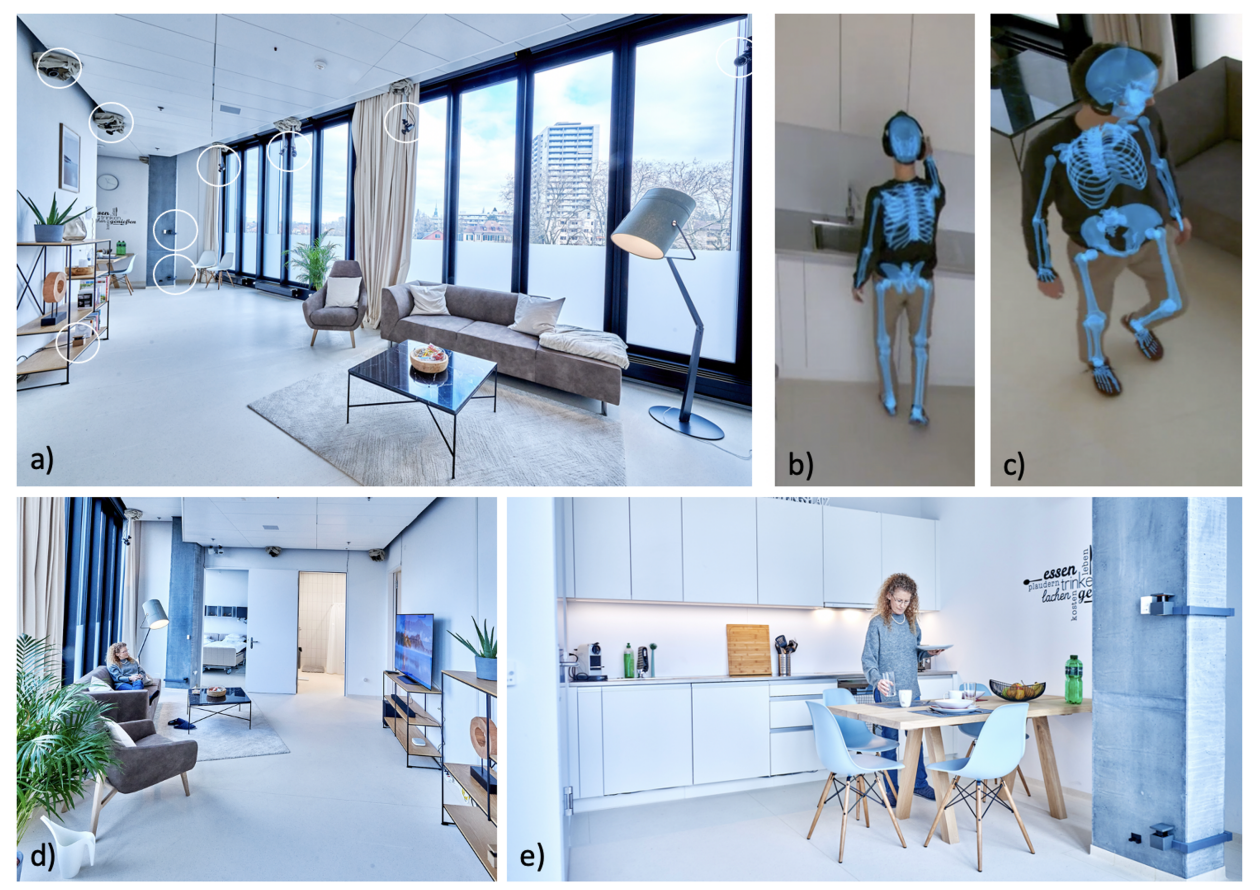

Figure 2. Marker-free motion tracking in the sensor-equipped apartment (NeuroTec Loft) University Hospital Inselspital. The living room (a,d) and the kitchen (e) are equipped with 13 cameras that allow to fit a 54-point skeleton model onto the test-person's body $(\mathbf{b}, \mathbf{c})$.

\section{Personalized Chronotherapy Is Important for the Future of Epilepsy Care}

Epilepsy is a periodic disorder of the brain that ranks among the most devastating neurological conditions, affecting all ages, including young and otherwise healthy people. Although the cyclical nature of seizures in epilepsy has been recognized since antiquity [18], the scientific study of these phenomena is only starting to emerge, in part because patientreported seizure burden is often inaccurate [19]. Furthermore, overt seizures are only the tip of the iceberg in epilepsy. It is now recognized that epileptic brain activity is present on an ongoing basis in most patients, without accompanying (obvious) symptoms. Although the pathological significance of such activity is still an area of active research, it can be used as a (digital) biomarker to monitor disease activity. However, the current practice of adjusting treatments in epilepsy still relies on self-reports of seizures at quarterly medical appointments, where patient's recall of intervening events is, at best, fuzzy due to the time elapsed. Thus, there is an unmet need for tighter monitoring of brain activity in epilepsy, which motivates the development of novel recording modalities, including wearables, smartwatches, headbands with integrated EEG electrodes and implantable EEG systems. The latter, despite the need for surgery, may represent a particularly attractive solution for patients who need continuous monitoring of brain activity to capture seizures that can occur at any moment.

Recently, using such a system, we have shown that seizures obey certain timing rules (Figure 3) that are individual, but also shared at the group level (so-called "seizure chronotypes"). Preferential seizure timing was found at three timescales, from most to least 
prevalent: circadian (90\%), multidien ( $60 \%$ ) and circannual ( $10 \%$ ) [20]. Multidien cycles of seizures can be further divided into 7-day, 15-day, 20-day and 30-day chronotypes. Relying on such patient-specific patterns of seizure recurrence, we were able to train statistical models to forecast seizure risk over the next cycle, up to three days in advance [21]. This confirms that seizures are not entirely random events and opens the way for timevarying therapies.

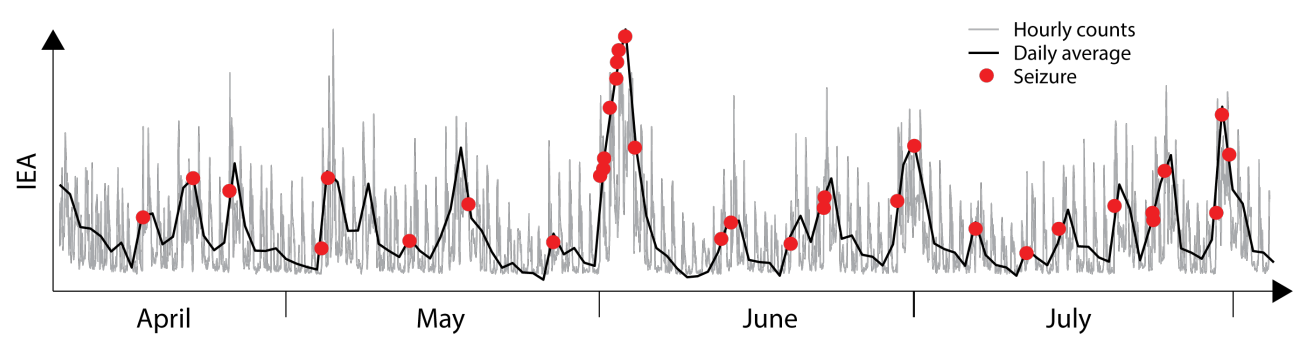

Figure 3. Ultra-long term recording of human brain activity with intracranial electrodes over months to years demonstrates that epileptic seizures do not occur randomly. The interictal epileptiform activity (IEA) - here quantified by a composite measure of EEG signal features—is characterized by multi-dien periodicities. Epileptic seizures are more likely to occur during times of increasing and/or increased IEA.

At NeuroTec, we use the most recently developed subscalp EEG recording devices [22], aiming to continuously monitor patients with epilepsy night and day to determine patientspecific seizure chronotypes, while at home, recordings from patients will be automatically scanned, using machine-learning algorithms. In addition, we will also take advantage of the sleep labs at NeuroTec to assess behavioral correlates of recorded nocturnal epileptic brain activity. In parallel, on-site measurements will be carried out, using transcranial magnetic stimulation combined with EEG (TMS-EEG) to assess the dynamics of cortical excitability, which is hypothesized to underlie the cyclical timing of seizures. In combining these approaches-implanted EEG, TMS-EEG and behavioral assessments-we will achieve a thorough level of understanding of individual manifestations of epilepsy and their chronicity. Precision, personalization and dynamical assessments are required to enable chronotherapy in epilepsy, which targets periods of heightened seizure risk. In this setting, the NeuroTec facilities are designed for comfortable access by patients, they have all the required state-of-the-art equipment, and they promote ease of integration of clinical care and research, which is notoriously hard to achieve in a hospital or clinic setting. In the long run, monitoring and probing the brain in individual patients with epilepsy will enable clinical trials of personalized and dynamical drug therapies, with a tight monitoring of drug-responsiveness, curtailing the long wait for the "next seizure". This proactive mode of data-driven adjustments to treatment is expected to increase quality of life, due to the better treatment outcome, focus on individual patients, and continued improvements of diagnostics and care based on the latest research findings. NeuroTec is at the forefront of this new model that uses continuous patient data as the driver of the diagnostic and treatment processes instead of insular appointments with the treating neurologist.

\section{Detecting Primary Generalized Epileptic Seizures by Less Obtrusive Technology}

Primary generalized seizures are characterized by the bi-hemispheric simultaneous onset of epileptiform signals and may be detected with only a few EEG electrodes applied to the scalp. Clinically, primary generalized seizures typically involve the sudden impairment of consciousness. As patients often do not realize or remember that they have had a primary generalized seizure, the treating physician is faced with incomplete or inaccurate information. Reliable long-term monitoring of primary generalized seizures with few scalp EEG electrodes is, therefore, expected to significantly improve personalized treatment by providing objective information about the rate and individual pattern of seizure occurrences on different time scales and thus promote chronotherapy as de- 
scribed in the preceding section. However, patient compliance is a key factor for success to achieve reliable long-term EEG monitoring. In the context of epilepsy patients, who still experience enormous levels of stigmatization [23], it is essential to provide an inconspicuous solution for recording brain activity to ensure patient adherence. Today, neither bulky multi-channel EEG devices nor consumer-grade EEG headbands found in the market satisfy this urgent demand. A project termed ULTEEM (ultra-long-term EEG monitoring), funded by the European Space Agency (ESA) and CSEM (Swiss center for electronics and microtechnology), set out to address this unmet medical need by placing the patients comfort at the center of the solution. The new proof-of-concept ULTEEM device, designed by CSEM, comprises two sensors that can be clipped onto any metallic frame of eyeglasses (Figure 4). Such a solution is only possible thanks to the unique technology developed by CSEM, allowing the connection of multiple sensors by a single wire, which does not even need to be shielded or insulated. Although the connection is simplified, the signal quality is not compromised. This crucial characteristic differentiates the ULTEEM device from consumer-grade EEG solutions, providing the neurologist with a signal quality equivalent to that of clinical-grade EEG devices. Applicable medical device standards (IEC 60601-1 and IEC 80601-2-26:2019) were followed during the design of the device. Using dry electrodes, the ULTEEM device acquires a 1-lead EEG between the two temples. The sensors are powered by a rechargeable battery and communicate with portable devices via the Bluetooth low energy protocol, which allows real-time visualization of the acquired data as well as downloading the data at a later time. The pilot study of the device was performed at NeuroTec EEG Labs with 10 healthy volunteers. The objectives of the evaluation were to assess the usability of the ULTEEM device in a clinical setting, as well as to benchmark the performance of the system with certified EEG devices. For each subject, the EEG signals were recorded for 20 min with ULTEEM and simultaneously with a certified EEG device, using gel electrodes for comparison. In addition to recordings during quiescence, the subjects were asked to perform activities, such as lateral eye movements, swallowing, and teeth clenching. All of the 10 subjects who participated in the study reported that the ULTEEM device was comfortable to wear, and they did not develop any headaches or feel discomfort during or after the recordings. Synchronized waveforms from the ULTEEM and reference EEG device were both qualitatively and quantitatively analyzed. Both types of signals for different time windows and for different activities were compared by an experienced neurologist, who concluded that the measurements acquired by the ULTEEM system match well with the measurements obtained with the reference device. Moreover, a similarity analysis was performed between two waveforms, using Pearson's correlation coefficient. For the cases where the subjects were instructed to perform lateral eye movements, a very strong correlation was observed (Pearson's $r>0.97)$. In the cases where the subjects were recorded during quiescence, akin to typical clinical recording conditions, the correlations were somewhat less pronounced but still strong (Pearson's $r>0.80$ ). In conclusion, a single-lead dry-electrode EEG device designed for ultra long-term monitoring of epilepsy patients was developed, and a pilot study using the device at NeuroTec EEG Labs yielded promising results. For the pilot study with healthy subjects, NeuroTec provided an ideal setting, where the subjects were able to be assessed by experienced neurologists from the Department of Neurology of Inselspital teaming up with engineers from CSEM to oversee the study and analyze the results. 


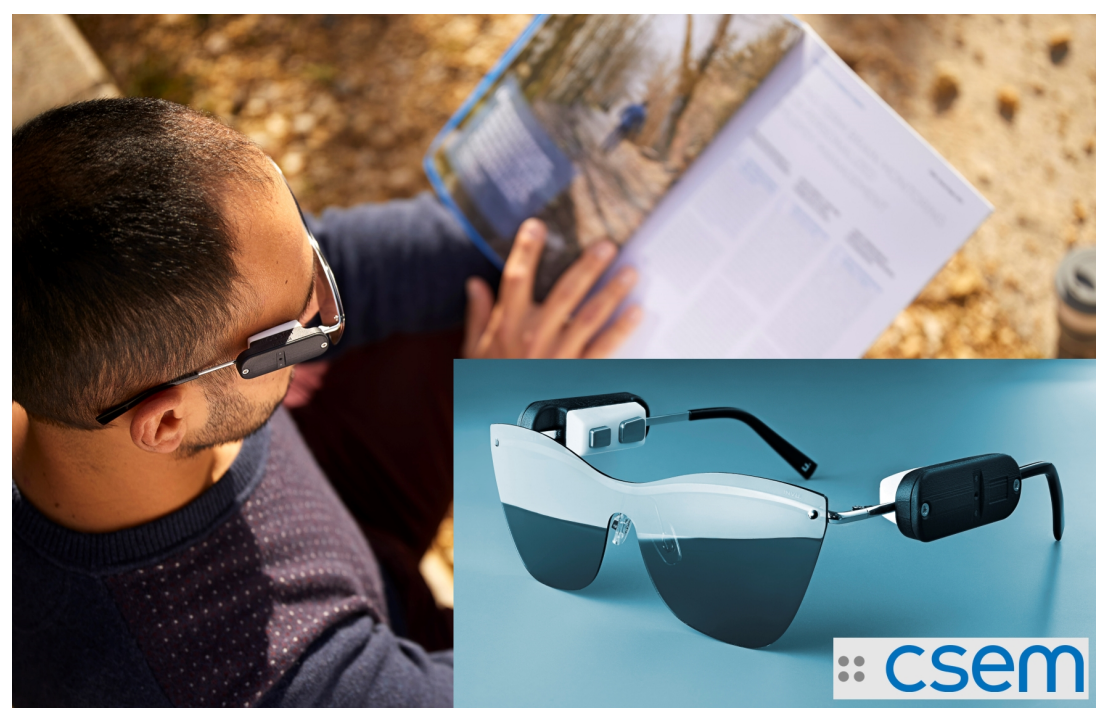

Figure 4. The ULTEEM recording system developed by CSEM (Swiss center for electronics and microtechnolgy) is integrated into glasses and thus minimizes obtrusiveness and stigmatization.

\section{Monitoring Sleep at-Home}

Sleep-wake-circadian changes (SWCD) have a bidirectional relationship with neurological and neuropsychiatric diseases. They can act as driving forces or modulators but also emerge as early symptoms [24-26]. To monitor sleep over extended periods, we want to test and validate a system that allows for unobtrusive, easy-to-use, and long-term in-home measuring of sleep and related relevant parameters. In neurological diseases, such as PD or Alzheimer's disease, sleep disorders may occur during the prodromal stages, even years before the emergence of cardinal symptoms $[27,28]$. As part of the standard clinical diagnostics, patients are referred to the sleep clinic for overnight polysomnography (PSG) [29] and additional daytime examinations during which parameters, such as brain activity by EEG, heart rate, muscular activity, eye movements, respiration rate, blood oxygen saturation, and general movements, are recorded and analyzed. Such snapshots provide the basis for a diagnosis but fail to detect fluctuations, periodicities, or subtle changes that can occur over time. Long-term monitoring is, therefore, paramount in detecting these changes or early indicators during the prodromal stages of neurodegenerative disease, when potential future treatments are expected to be most effective. This long-term monitoring in the hospital is not feasible for multiple reasons: high cost, unnatural environment for the patients, and the highly obtrusive character of the currently used devices, which might negatively affect sleep and thus, the data quality.

With advances in wireless technologies, new and less obtrusive wearable and ambient (smart objects with wireless connectivity) sensing devices for assessing sleep have been developed (e.g., pressure mattresses, radar and infrared sensors). Several studies have shown a high correlation between such wearable and ambient devices and the gold standard PSG $[30,31]$. To date, studies have often compared only one single device at a given time to PSG in terms of accuracy, and recruited only healthy participants, resulting in a lack of a gold standard on two levels: first, each wearable or ambient device records specific types of data, but it remains to be determined what combination of sensing devices may optimize recording capabilities; second, studies are lacking to validate such devices in a medical setting with patients with sleep disorders. Finally, such a sensor network would provide a means for long-term at-home monitoring. This is the starting point for our sleep monitoring study, funded by the Interfaculty Research Cooperation (IRC) "Decoding Sleep" of the University of Bern, to be conducted at NeuroTec. Most physiological parameters can be assessed by different sensing technologies. For example, the breathing rate may be quantified with a flow cannula placed in front of the nose, where the actual airflow is measured, but it can also be determined by the assessment of thoracic 
movements, or even be derived from electrocardiographic (ECG) signals [32]. While those technologies have their strengths and weaknesses (including movement artifacts as a common weakness), the combination of several technologies with partial redundancy of data might be the best approach to optimize measurements and minimize the risk of information loss. A second project within NeuroTec will study patients with chronic excessive daytime sleepiness and is known as the Swiss Primary Hypersomnolence and Narcolepsy Cohort Study (SPHYNCS). For this study, biomarkers to differentiate the causes of chronic sleepiness will be explored and will include diagnostic sleep testing, biological sampling, questionnaires and a long-term wearable device [33]. These two studies represent a new close collaboration between SWEZ and NeuroTec.

The novelty and strength of our approach is the combination of multiple ambient and wearable sensors into a network (Figure 5) installed in a NeuroTec sleep room to test if this unobtrusive sensor network succeeds where any single sensor currently fails to compare to the gold standard PSG in terms of data quality and accuracy. Patients with sleep disorders will be examined simultaneously with PSG and the multidimensional sensor network.

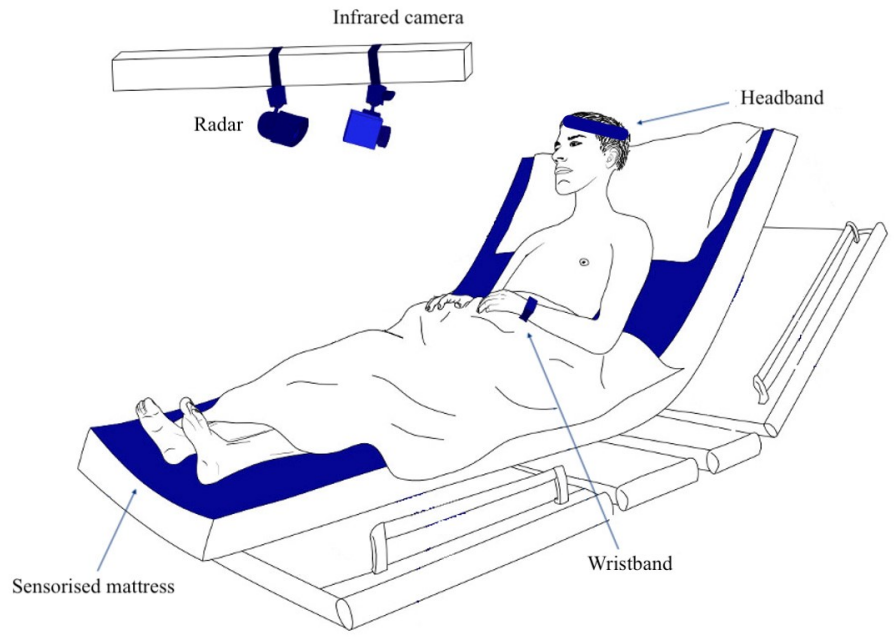

Figure 5. The unobtrusive multidimensional sensor network comprises contactless technologies, such as radar and infrared ("ambient" devices). The subject lies on a mattress with integrated pressure sensors, wearing a smart wristband and a headband with EEG electrodes ("wearables").

We aim to determine how these unobtrusive technologies can be combined into a sensor network with the ultimate goal (in future phases) of partially or fully replacing PSG to reduce obtrusiveness and thus to improve the comfort of patients during the assessment. If successful, it will enable home monitoring of patients without the need for technical support.

Based on the outcome of this sleep monitoring study (highest accuracy and reliability, user-friendliness, and least obtrusive character), we will select a final and smaller set of ambient and wearable sensors. This final set will then be further tested in a small cohort for long-term at-home monitoring, first at our NeuroTec Loft and then at the actual patient's home. Once available, this system would allow for the extended monitoring of patients with neurological diseases. Examples could be assessing the onset and evolution of REM behavior disorder in patients with synucleinopathies [34], the onset of sleep-wake-circadian disturbances in Alzheimer's disease [35], or the monitoring of patients with disorders that belong to the narcolepsy borderland [33]. However, one could also imagine a future where patients receive a kit with those sensors to be installed in their homes, even before a consultation with their sleep physician. This way, all relevant sleep parameters would be 
already known in the first consultation, providing a more enhanced basis for an accelerated diagnostic workup. Thus, the sleep monitoring study on the sensor network conducted at NeuroTec represents the launchpad for personalized sleep medicine in the patient's home.

\section{Computational Modeling and Machine Learning for Data Analysis}

In parallel to the development and validation of novel recording technologies, NeuroTec is also focusing on the development of novel methods for data analysis. Given that NeuroTec has the capacities for long-term EEG/sensor recordings, as well as for recordings of highdensity EEG, it becomes imperative to develop technologies to analyze the resulting data.

Computational modeling and machine learning approaches are increasingly used in most fields to facilitate the analysis of complex data sets. The field of computer vision has seen tremendous progress with the introduction of deep learning algorithms, which have led to unprecedented performance in various classification tasks [36]. However, the use of deep learning techniques in neuroscience and, in particular, EEG research remains limited. Two major challenges impede the more systematic use of these algorithms: the first is the limited amount of data, and the second is the interpretability. Deep neural networks need a substantial amount of data to learn from, in order to optimize their parameters without overfitting, i.e., fine-tuning to the input data, while being unable to generalize to new samples. This is typically not an issue for the field of computer vision, where large data sets with hundreds of thousands of images are available. By contrast, the available data in the field of EEG research remain limited to a few hundreds of repetitions per participant, due to practical limitations, such as fatigue or time resources [37]. This limitation in data collection may result in overfitting of most available neural networks. In our work, we are developing novel deep learning pipelines to augment EEG data sets in order to train deep neural networks without overfitting. Another direction of research that we are pursuing is that of interpretability in the field of deep learning. Understanding which features of a given data set contribute to the output of a neural network is crucial for introducing deep learning algorithms in basic and clinical research [38]. One main focus of research at NeuroTec is to develop algorithms that are not only powerful in classifying data, but also interpretable. In our ongoing endeavors, we are examining the effects of network architecture in the networks' performance, and also in the interpretability of the extracted features. Additionally, we are assessing the effects of bias in biomedical data sets that are oftentimes used to train neural networks [39,40]. Future work in this direction can build bridges to clinical neuroscience research with tools from the field of deep learning to classify disease phenotypes and establish markers of disease progression, with particular emphasis on sleep research [41].

To complement methodological developments for the field of EEG research, we are additionally designing novel experimental paradigms that take advantage of the high density EEG setup and sleep laboratory of NeuroTec. Our main focus is in consciousness research, and in particular in studying the neural functions that are preserved in conditions of reduced conscious access to the environment, such as during sleep. During sleep, conscious perception of the environment fades away. Nevertheless, despite a temporary loss of consciousness, the human brain continues to process sensory information from its surroundings. Sounds can reach the sensory areas of the brain and are processed during sleep, without our overt awareness. The processing of sensory information in the absence of consciousness has also been observed in pathological loss of consciousness, such as during coma $[40,42]$. Interestingly, the progression of the neural discrimination of auditory stimuli in the first days of coma has been shown to be an indicator of post-anoxic coma patients' chances of regaining consciousness [43]. Despite the clinical relevance that the neural discrimination of auditory stimuli has in relation to consciousness, its neural substrates remain underexplored.

In our work, we are combining computational modeling approaches with experimental techniques, such as scalp and intracranial EEG recordings [44], to study the neural substrates that allow us to process and discriminate sounds from the environment in re- 
duced consciousness conditions. Improving our understanding of how sensory predictive functions are altered by the presence or loss of consciousness could have applications in clinical research. To date, one main challenge in consciousness research is to assess the levels of consciousness of an unresponsive patient. Because of their subjective nature, levels of consciousness are prone to misclassification [45]. Patients with disorders of consciousness are often non-responsive to their environment and it is thus hard, if not impossible, to perform behavioral clinical tests on them. Because of their fluctuating levels of consciousness, and limited abilities for communication, a misdiagnosis results in up to $40 \%$ of cases [45]. To overcome the limitations of behavioral tests, EEG-based approaches have been introduced to assess levels of consciousness in a more objective way. Several tests, mainly relying on event-related potentials (ERPs), examine the presence or absence of EEG responses to sensory stimuli, such as the patient's own name [46], or the detection of auditory regularities [47]. However, these approaches have several drawbacks: (a) they assume a similarity between neural responses in healthy individuals and patients with disorders of consciousness; (b) they are often based on the absence of evidence such as the absence of a neural EEG response-which might be confounded by low statistical power; and (c) they often do not have a ground truth for assessing levels of consciousness, as several of these approaches have not been assessed in controlled conditions. There is, thus, a strong need for novel, controlled experimental paradigms and automated tools that can assist clinical diagnoses of levels of consciousness in a patient-specific way. As an alternative to traditional ERP-based analyses, machine learning approaches can be used. These approaches extract, in a data-driven way, patterns of EEG activity that differ between conditions of interest, and use those patterns to classify EEG signals [48]. The advantage of machine-learning approaches over ERP-based analyses is that they can model EEG data at the single-participant level, which is particularly relevant for the case of clinical studies, where inter-individual variability is high [49]. Our work at NeuroTec, combining high-density EEG recordings in sleep and wakefulness with machine learning approaches, aims at advancing our understanding of alterations in neural functions following loss of consciousness. This line of research could assist with future clinical diagnosis and prognostication of patients with disorders of consciousness.

\section{From Diagnostics to at-Home Therapies: The Case of Non-Invasive Brain Stimulation}

Pharmacological interventions represent only a partial solution for the treatment of disorders of the central nervous system, due to the absence of effective molecules, treatment resistance, and tolerance issues. A promising, complementary strategy is the more direct modulation of brain activity by delivering electromagnetic energy. The last twenty years have seen a tremendous growth of research and clinical applications of non-invasive brain stimulation. In particular, transcranial magnetic stimulation (TMS) and transcranial electrical stimulation (tES) are used in the research lab to (1) investigate the causal role of spatio-temporal activity patterns in cognition and behavior and (2) attempt to restore disordered neuronal activity in disease states. Yet, despite the extremely favorable safety profile of non-invasive brain stimulation [50,51], fundamental questions remain unaddressed. First, only a minority of studies have asked whether the investigated brain stimulation paradigm successfully engages the network target (i.e., has the desired specific effect on the targeted brain activity). Importantly, the interpretation of an association between a behavioral metric and a specific stimulation paradigm is challenging in the absence of neurophysiological or neuroimaging data on target engagement [52]. Second, most of the brain stimulation research is performed in the research laboratory, partially due to equipment constraints (for example, a TMS device requires a dedicated lab space and experienced technicians to operate it), safety concerns in the case of incorrect use, such as burns caused by inappropriately applied stimulation electrodes for $\mathrm{tES}$, and heterogeneity introduced in research performed outside of the research lab. This represents a missed opportunity since bringing stimulation neurotechnology to the patient's home for treatment will close the last mile in neurology and offer vastly increased treatment access at 
the population level. By addressing these two main challenges (measuring target engagement and studying neurotechnology for home use), NeuroTec positions itself at the leading edge of non-invasive brain stimulation with the promise of becoming a unique hub that integrates medicine, neuroscience, and engineering expertise to change patients' lives.

Traditionally, brain stimulation was conceptualized as delivering energy to specific brain areas that are affected in disease states. One highly successful example is (invasive) deep brain stimulation for the treatment of Parkinson's disease (see above). However, complex behavior and disease states often do not map onto specific brain areas but rather emerge from the (nonlinear) network interactions of numerous brain circuits and areas. Coordination and communication in such networks are mediated by the temporal synchronization of rhythmic patterns in macroscopic electric brain activity. The growing insight that these signals, which can be routinely measured by EEG, are at the heart of cognition and behavior caused a rebirth of EEG, which had been temporarily marginalized by the arrival of magnetic resonance imaging. EEG is non-invasive, can be deployed in and outside the lab (see above for innovation in this space at NeuroTec), and captures the activity dynamics of brain networks with high temporal resolution. Advances in analysis strategies provide quantitative insights about network interactions and directed information flow. The last ten years have seen a growing interest in modulating these rhythmic activity patterns to establish their causal role in behavior and as a potential treatment for disorders associated with altered oscillation patterns (so-called "oscillopathies"). Two techniques are currently used for this purpose. The first is using TMS for modulating oscillations by adjusting the stimulation pulse frequency to match the targeted oscillation [53]. TMS boasts high spatial resolution but has several disadvantages that prevents it from being the neurotechnology solution for closing the last mile in neurology (e.g., cost, energy consumption, size and weight). In contrast, transcranial alternating current stimulation (tACS, $[54,55]$ (Figure 6)) is a cost-effective and portable technology that is battery-powered, thereby being well positioned to become a prominent player as an at-home therapeutic tool for neurological and psychiatric disorders [56,57]. tACS applies a weak electric current (typical amplitude: $2 \mathrm{~mA})$ to the scalp, which results in a weak electric field $(<1 \mathrm{~V} / \mathrm{m})$ in the brain. After some initial skepticism as to whether such a weak perturbation that changes the membrane voltage by less than $1 \mathrm{mV}$ can indeed modulate network dynamics, it has become clear that neural oscillations are highly susceptible to even very weak periodic perturbations that are designed to match the endogenous frequency of brain oscillations via a well-established principle from physics referred to as the Arnold tongue [58].

Initial clinical trials have demonstrated that tACS can modulate physiological and pathological oscillations in a targeted manner by entrainment and that stimulation paradigms as short as five days can induce changes in brain activity that persist for weeks [56]. Yet, the durability of both neural and clinical effects remains a concern that can be addressed by repeat stimulation, which makes a clinic-based approach cumbersome and costly. Several pilot studies support the safety of paradigms for up to 20 weekly tACS sessions [59]. Yet, the next step of deploying this technology at-home in a scientifically rigorous way remains a key obstacle for the advancement of this technology. The NeuroTec Loft is an ideal environment to close this last mile by studying tACS (and, in the future, other portable and targeted neurostimulation technologies) in a setup that exhibits the main features of what home use would entail, while still offering the research infrastructure- such as high-density EEG and advanced video monitoring, typically only available in research lab spaces-that lacks the external validity offered by the NeuroTec Loft, which mimics an apartment. 


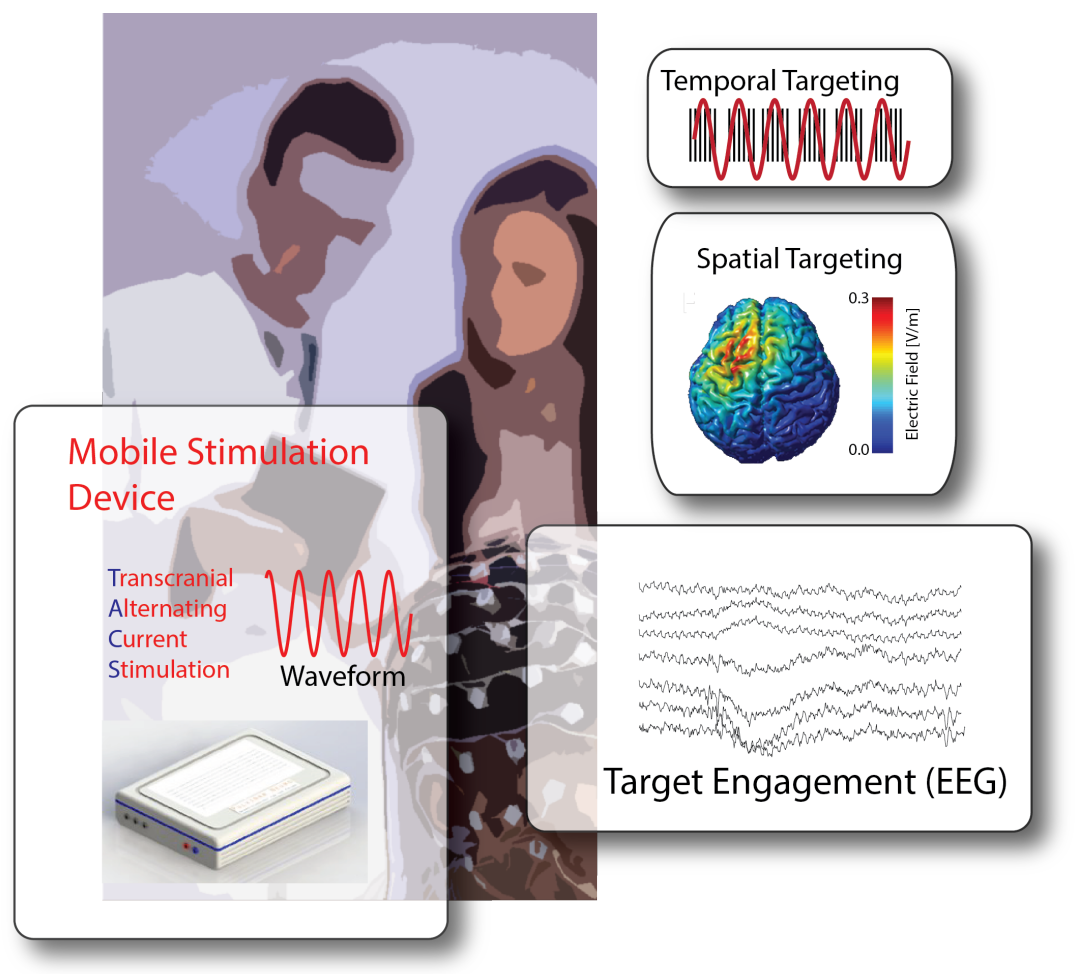

Figure 6. Non-invasive brain stimulation by transcranial alternating current stimulation (tACS) modulates brain rhythms by entrainment (temporal targeting). The spatial spread of the electric field is determined by the montage of the electrodes (spatial targeting). The stimulation waveform is a sine wave tuned in frequency to the targeted network oscillation. Target engagement (and closed-loop stimulation) is based on EEG. The tACS technology is safe, lightweight, easy to apply, cost effective and, therefore, ideal for future at-home use.

NeuroTec is working with the Carolina Center for Neurostimulation at the University of North Carolina and Pulvinar Neuro, a brain stimulation university spin-off company supported by the National Institutes of Health, to prepare for leveraging NeuroTec as a collaborative test location to evaluate device technologies and perform studies that provide the beach head toward at-home studies. Currently, Pulvinar Neuro is developing a cloudintegrated stimulation platform that allows multisite trials and eventually at-home stimulation by cloud-controlled stimulation and study management. Next steps could include the testing of closed-loop stimulation paradigms developed for the targeting of transient neural oscillations during sleep [60].

\section{Summary and Outlook}

We have here described the first-generation projects carried out at the recently founded NeuroTec sitem-insel. These sample projects illustrate that the research and development at NeuroTec has implications for a large set of disorders and disease states. This spectrum ranges from neurodegenerative disorders, such as Parkinson's or Alzheimer's disease, to epilepsy, sleep-wake-circadian disturbances, stroke and even psychiatric conditions. However, despite this multitude of disorders, the research efforts at NeuroTec are tightly interrelated, due to the strong overlap of applied technologies and methods (Figure 7). Yet, the coherence of the NeuroTec approach runs deeper than being merely methodical because many —if not all—of the aforementioned disorders are also bound together through their conceptualization as disorders with shared (network) pathophysiology. To provide one important example, recent studies strongly indicate that both epileptic seizures and sleep-wake-circadian disturbances might promote Alzheimer's disease $[28,61]$ and thus offer potential new treatment strategies for this devastating condition that looms over the progressively aging humankind. We propose that progress in tackling such challenges 
requires exactly the kind of ecosystem for the collaborations offered by NeuroTec, liberated from the silos of academic departments and from the constraints of established paradigms. Developing new diagnostics and treatments for brain disorders has remained a humbling challenge despite the numerous advances in neuroscience research over the last decade. The key to unlocking the translational benefits of basic science for neurological disorders is to provide a setting that attracts clinicians and clinician-scientists who are passionate about pushing boundaries and innovating through collaborations. NeuroTec provides a unique opportunity for the interdisciplinary and interprofessional training of the next generation of clinicians, engineers, MD-PhDs, and data and basic scientists. Crucial educational aspects will include sensitizing health professionals for privacy and data safety [62] and to inform about risks and chances of invoking emerging technologies, such as blockchain [63], edge computing [64] or combinations thereof [65], to address these issues.

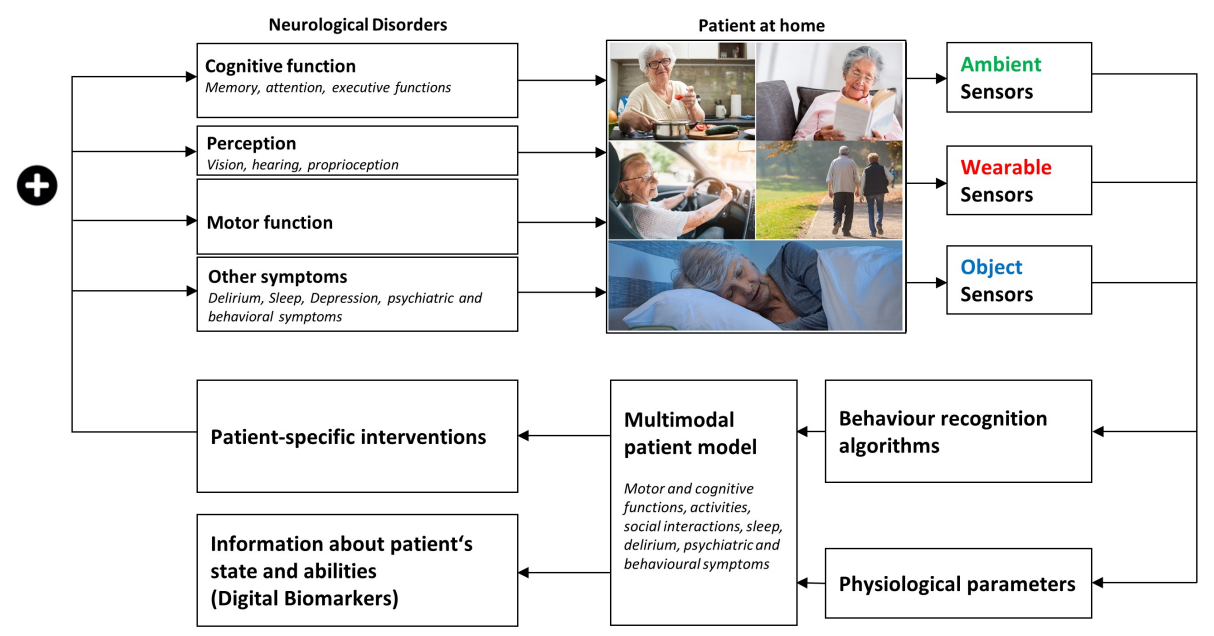

Figure 7. Overview of the general approach pursued at NeuroTec: novel sensor technologies are applied to yield a combination of ambient context information with specific information from body mounted sensors. These data are then used to develop a multimodal patient model that enables individualized interventions.

One further common thread in the institutional DNA of NeuroTec is the focus on personalized medicine. By putting patients first across the entire life cycle of projects in both basic and clinical science, we are creating a culture that elevates technology and methods to improve the quality of life and outcome in patients. The goal is to make diagnostic and treatment procedures not only better, but also more tolerable because they can be performed in the privacy of the patient's home and, in the future, also in other natural habitats, as well as during other daily activities, such as commuting [66] or doing sports [67]. Finally, for the next generation of neurotechnology and digital biomarkers to reach patients, ultimately, commercialization is required. NeuroTec has structurally addressed this need through its framework that allows different flavors of public-private collaborations and partnerships to help companies push the discoveries out of the lab into the hands of clinicians and patients.

We hope and expect that NeuroTec sitem-insel will develop into an internationally renowned platform that promotes innovative and effective collaboration between scientists, engineers and clinicians to develop the future technologies that we will need to bring diagnostics and therapies to the home of the patient, thus helping to close the last mile in neurology. 
Author Contributions: Conceptualization: K.A.S., T.N. and F.F.; all authors contributed to writing. All authors have read and agreed to the published version of the manuscript.

Funding: This research was financed in parts by the Swiss National Science Foundation Bridge Discovery Grant "Emotion in the loop'-a step towards a comprehensive closed-loop deep brain stimulation in Parkinson's disease", and the Swiss National Science Foundation Grant "Swiss Primary Hypersomnolence and Narcolepsy Cohort Study"-No. 185362.

Institutional Review Board Statement: Not applicable.

Informed Consent Statement: Not applicable.

Data Availability Statement: Data are contained within the article.

Conflicts of Interest: F.F. is the founder, shareholder, and chief science officer of Pulvinar Neuro LLC. M.O.B. reports the personal fees and grants from the Wyss Center for neurotechnology in Geneva, and has a patent application pending under the Patent Cooperation Treaty (62665486). K.A.S. reports the personal fees from the Wyss Center for neurotechnology in Geneva and from WoodWelding SA in Stansstad, Switzerland. He also is a medical consultant for Pulvinar Neuro LLC. The other authors declare no conflict of interest.

\section{References}

1. Baud, M.O.; Proix, T.; Rao, V.R.; Schindler, K. Chance and risk in epilepsy. Curr. Opin. Neurol. 2020, 33, 163-172. [CrossRef] [PubMed]

2. Krack, P.; Volkmann, J.; Tinkhauser, G.; Deuschl, G. Deep brain stimulation in movement disorders: From experimental surgery to evidence-based therapy. Mov. Disord. 2019, 34, 1795-1810. [CrossRef]

3. Torkildsen, Ø.; Linker, R.A.; Sesmero, J.M.; Fantaccini, S.; de la Rosa, R.S.; de Seze, J.; Duddy, M.; Chan, A. Living with secondary progressive multiple sclerosis in Europe: Perspectives of multiple stakeholders. Neurodegener. Dis. Manag. 2021, 11, 9-19. [CrossRef] [PubMed]

4. Tarnanas, I.; Laskaris, N.; Tsolaki, M.; Muri, R.; Nef, T.; Mosimann, U.P. On the comparison of a novel serious game and electroencephalography biomarkers for early dementia screening. In Advances in Experimental Medicine and Biology; Springer International Publishing: Berlin/Heidelberg, Germany, 2014; pp. 63-77. [CrossRef]

5. Klaffke, S.; Staedt, J. Sundowning and circadian rhythm disorders in dementia. Acta Neurol. Belg. 2006, 106, 168-175. [PubMed]

6. Burish, M.J.; Chen, Z.; Yoo, S.H. Cluster Headache Is in Part a Disorder of the Circadian System. JAMA Neurol. 2018, 75, 783. [CrossRef]

7. Wehr, T.A. Bipolar mood cycles and lunar tidal cycles. Mol. Psychiatry 2018, 23, 923-931. [CrossRef] [PubMed]

8. Facchin, L.; Schöne, C.; Mensen, A.; Bandarabadi, M.; Pilotto, F.; Saxena, S.; Libourel, P.A.; Bassetti, C.L.; Adamantidis, A.R. Slow waves promote sleep-dependent plasticity and functional recovery after stroke. J. Neurosci. 2020, 40, 8637-8651. [CrossRef] [PubMed]

9. Baud, M.O.; Kleen, J.K.; Mirro, E.A.; Andrechak, J.C.; King-Stephens, D.; Chang, E.F.; Rao, V.R. Multi-day rhythms modulate seizure risk in epilepsy. Nat. Commun. 2018, 9, 88. [CrossRef]

10. Ten Brinke, T.R.; Odekerken, V.J.J.; Dijk, J.M.; van den Munckhof, P.; Schuurman, P.R.; de Bie, R.M.A. Directional Deep Brain Stimulation: First experiences in centers across the globe. Brain Stimul. 2018, 11, 949-950. [CrossRef] [PubMed]

11. Meidahl, A.C.; Tinkhauser, G.; Herz, D.M.; Cagnan, H.; Debarros, J.; Brown, P. Adaptive Deep Brain Stimulation for Movement Disorders: The Long Road to Clinical Therapy. Mov. Disord. 2017, 32, 810-819. [CrossRef] [PubMed]

12. Tinkhauser, G.; Pogosyan, A.; Tan, H.; Herz, D.M.; Kühn, A.A.; Brown, P. Beta burst dynamics in Parkinson's disease OFF and ON dopaminergic medication. Brain 2017, 140, 2968-2981. [CrossRef]

13. Bouthour, W.; Mégevand, P.; Donoghue, J.; Lüscher, C.; Birbaumer, N.; Krack, P. Biomarkers for closed-loop deep brain stimulation in Parkinson disease and beyond. Nat. Rev. Neurol. 2019, 15, 343-352. [CrossRef]

14. Little, S.; Brown, P. Debugging Adaptive Deep Brain Stimulation for Parkinson's Disease. Mov. Disord. 2020, $35,555-561$. [CrossRef] [PubMed]

15. Ossig, C.; Antonini, A.; Buhmann, C.; Classen, J.; Csoti, I.; Falkenburger, B.; Schwarz, M.; Winkler, J.; Storch, A. Wearable sensor-based objective assessment of motor symptoms in Parkinson's disease. J. Neural Transm. 2015, 123, 57-64. [CrossRef]

16. Rovini, E.; Maremmani, C.; Cavallo, F. How wearable sensors can support Parkinson's disease diagnosis and treatment: A systematic review. Front. Neurosci. 2017, 11, 555. [CrossRef] [PubMed]

17. Schütz, N.; Saner, H.; Rudin, B.; Botros, A.; Pais, B.; Santschi, V.; Buluschek, P.; Gatica-Perez, D.; Urwyler, P.; Marchal-Crespo, L.; et al. Validity of pervasive computing based continuous physical activity assessment in community-dwelling old and oldest-old. Sci. Rep. 2019, 9, 9662. [CrossRef]

18. Magiorkinis, E.; Sidiropoulou, K.; Diamantis, A. Hallmarks in the history of epilepsy: From antiquity till the twentieth century. In Novel Aspects on Epilepsy; InTech: London, UK, 2011. [CrossRef] 
19. Elger, C.E.; Hoppe, C. Diagnostic challenges in epilepsy: Seizure under-reporting and seizure detection. Lancet Neurol. 2018, 17, 279-288. [CrossRef]

20. Leguia, M.G.; Andrzejak, R.G.; Rummel, C.; Fan, J.M.; Mirro, E.A.; Tcheng, T.K.; Rao, V.R.; Baud, M.O. Seizure cycles in focal epilepsy. JAMA Neurol. 2021, 78, 454-463. [CrossRef]

21. Proix, T.; Truccolo, W.; Leguia, M.G.; Tcheng, T.K.; King-Stephens, D.; Rao, V.R.; Baud, M.O. Forecasting seizure risk in adults with focal epilepsy: A development and validation study. Lancet Neurol. 2021, 20, 127-135. [CrossRef]

22. Duun-Henriksen, J.; Baud, M.; Richardson, M.P.; Cook, M.; Kouvas, G.; Heasman, J.M.; Friedman, D.; Peltola, J.; Zibrandtsen, I.C.; Kjaer, T.W. A new era in electroencephalographic monitoring? Subscalp devices for ultra-long-term recordings. Epilepsia 2020, 61, 1805-1817. [CrossRef] [PubMed]

23. Eichenwald, K. A Mind Unraveled: A Memoir; Ballantine Books: New York, NY, USA, 2018.

24. Bassetti, C.L.; Ferini-Strambi, L.; Brown, S.; Adamantidis, A.; Benedetti, F.; Bruni, O.; Cajochen, C.; Dolenc-Groselj, L.; Ferri, R.; Gais, S.; et al. Neurology and psychiatry: Waking up to opportunities of sleep: State of the art and clinical/research priorities for the next decade. Eur. J. Neurol. 2015, 22, 1337-1354. [CrossRef]

25. Leng, Y.; Musiek, E.S.; Hu, K.; Cappuccio, F.P.; Yaffe, K. Association between circadian rhythms and neurodegenerative diseases. Lancet Neurol. 2019, 18, 307-318. [CrossRef]

26. Rakusa, M.; Sieminski, M.; Rakusa, S.; Falup-Pecurariu, C.; Fronzcek, R.; Hidalgo, H.; Muntean, M.L.; Pijpers, A.; Cochen De Cock, V.; Pizza, F.; et al. Awakening to sleep disorders in Europe: Survey on education, knowledge, and treatment competence of European residents and neurologists. Eur. J. Neurol. 2021. [CrossRef]

27. Postuma, R.B.; Gagnon, J.F.; Vendette, M.; Montplaisir, J.Y. Markers of neurodegeneration in idiopathic rapid eye movement sleep behaviour disorder and Parkinson's disease. Brain 2009, 132, 3298-3307. [CrossRef]

28. Winer, J.R.; Mander, B.A.; Kumar, S.; Reed, M.; Baker, S.L.; Jagust, W.J.; Walker, M.P. Sleep disturbance forecasts $\beta$-amyloid accumulation across subsequent years. Curr. Biol. 2020, 30, 4291-4298. [CrossRef]

29. Rundo, J.V.; Downey, R. Polysomnography. In Clinical Neurophysiology: Basis and Technical Aspects; Elsevier: Amsterdam, The Netherlands, 2019; pp. 381-392. [CrossRef]

30. Mantua, J.; Gravel, N.; Spencer, R. Reliability of sleep measures from four personal health monitoring devices compared to research-based actigraphy and polysomnography. Sensors 2016, 16, 646. [CrossRef] [PubMed]

31. Roberts, D.M.; Schade, M.M.; Mathew, G.M.; Gartenberg, D.; Buxton, O.M. Detecting sleep using heart rate and motion data from multisensor consumer-grade wearables, relative to wrist actigraphy and polysomnography. Sleep 2020, 43, zsaa045. [CrossRef]

32. Massaroni, C.; Nicolò, A.; Presti, D.L.; Sacchetti, M.; Silvestri, S.; Schena, E. Contact-based methods for measuring respiratory rate. Sensors 2019, 19, 908. [CrossRef] [PubMed]

33. Dietmann, A.; Wenz, E.; van der Meer, J.; Ringli, M.; Warncke, J.D.; Edwards, E.; Schmidt, M.H.; Bernasconi, C.A.; Nirkko, A.; Strub, M.; et al. The Swiss Primary Hypersomnolence and Narcolepsy Cohort study (SPHYNCS): Study protocol for a prospective, multicentre cohort observational study. J. Sleep Res. 2021, e13296. [CrossRef]

34. Fereshtehnejad, S.M.; Yao, C.; Pelletier, A.; Montplaisir, J.Y.; Gagnon, J.F.; Postuma, R.B. Evolution of prodromal Parkinson's disease and dementia with Lewy bodies: A prospective study. Brain 2019, 142, 2051-2067. [CrossRef] [PubMed]

35. Musiek, E.S.; Bhimasani, M.; Zangrilli, M.A.; Morris, J.C.; Holtzman, D.M.; Ju, Y.S. Circadian Rest-Activity Pattern Changes in Aging and Preclinical Alzheimer Disease. JAMA Neurol. 2018, 75, 582-590. [CrossRef]

36. Goodfellow, I.; Bengio, Y.; Courville, A. Deep Learning; MIT Press: Cambridge, MA, USA, 2016. Available online: http: //www.deeplearningbook.org (accessed on 1 June 2021).

37. Williams, J.M.; Samal, A.; Rao, P.K.; Johnson, M.R. Paired trial classification: A novel deep learning technique for MVPA. Front. Neurosci. 2020, 14, 417. [CrossRef]

38. Mehrer, J.; Spoerer, C.J.; Kriegeskorte, N.; Kietzmann, T.C. Individual differences among deep neural network models. Nat. Commun. 2020, 11, 5725. [CrossRef]

39. Tzovaras, B.G.; Tzovara, A. The Personal Data Is Political. In Philosophical Studies Series; Springer International Publishing: Berlin/Heidelberg, Germany, 2019; pp. 133-140. [CrossRef]

40. Tzovara, A.; Simonin, A.; Oddo, M.; Rossetti, A.O.; Lucia, M.D. Neural detection of complex sound sequences in the absence of consciousness. Brain 2015, 138, 1160-1166. [CrossRef] [PubMed]

41. Dietmann, A.; Gallino, C.; Wenz, E.; Mathis, J.; Bassetti, C.L.A. Multiple sleep latency test and polysomnography in patients with central disorders of hypersomnolence. Sleep Med. 2021, 79, 6-10. [CrossRef]

42. Cossy, N.; Tzovara, A.; Simonin, A.; Rossetti, A.O.; Lucia, M.D. Robust discrimination between EEG responses to categories of environmental sounds in early coma. Front. Psychol. 2014, 5, 155.10.3389/fpsyg.2014.00155. [CrossRef] [PubMed]

43. Tzovara, A.; Rossetti, A.O.; Juan, E.; Suys, T.; Viceic, D.; Rusca, M.; Oddo, M.; Lucia, M.D. Prediction of awakening from hypothermic postanoxic coma based on auditory discrimination. Ann. Neurol. 2016, 79, 748-757. [CrossRef] [PubMed]

44. Johnson, E.L.; Kam, J.W.Y.; Tzovara, A.; Knight, R.T. Insights into human cognition from intracranial EEG: A review of audition, memory, internal cognition, and causality. J. Neural Eng. 2020, 17, 051001. [CrossRef]

45. Schnakers, C.; Vanhaudenhuyse, A.; Giacino, J.; Ventura, M.; Boly, M.; Majerus, S.; Moonen, G.; Laureys, S. Diagnostic accuracy of the vegetative and minimally conscious state: Clinical consensus versus standardized neurobehavioral assessment. BMC Neurol. 2009, 9, 35. [CrossRef] 
46. Kempny, A.M.; James, L.; Yelden, K.; Duport, S.; Farmer, S.F.; Playford, E.D.; Leff, A.P. Patients with a severe prolonged disorder of consciousness can show classical EEG responses to their own name compared with others' names. Neuroimage Clin. 2018, 19,311-319. [CrossRef] [PubMed]

47. Bekinschtein, T.A.; Dehaene, S.; Rohaut, B.; Tadel, F.; Cohen, L.; Naccache, L. Neural signature of the conscious processing of auditory regularities. Proc. Natl. Acad. Sci. USA 2009, 106, 1672-1677. [CrossRef]

48. Grootswagers, T.; Wardle, S.G.; Carlson, T.A. Decoding dynamic brain patterns from evoked responses: A tutorial on multivariate pattern analysis applied to time series neuroimaging data. J. Cogn. Neurosci. 2017, 29, 677-697. [CrossRef]

49. Lucia, M.D.; Tzovara, A. Decoding auditory EEG responses in healthy and clinical populations: A comparative study. J. Neurosci. Methods 2015, 250, 106-113. [CrossRef]

50. Antal, A.; Alekseichuk, I.; Bikson, M.; Brockmöller, J.; Brunoni, A.; Chen, R.; Cohen, L.; Dowthwaite, G.; Ellrich, J.; Flöel, A.; et al. Low intensity transcranial electric stimulation: Safety, ethical, legal regulatory and application guidelines. Clin. Neurophysiol. 2017, 128, 1774-1809. [CrossRef] [PubMed]

51. Matsumoto, H.; Ugawa, Y. Adverse events of tDCS and tACS: A review. Clin. Neurophysiol. Pract. 2017, 2, 19-25. [CrossRef] [PubMed]

52. Kurmann, R.; Gast, H.; Schindler, K.; Fröhlich, F. Rational design of transcranial alternating current stimulation: Identification, engagement, and validation of network oscillations as treatment targets. Clin. Transl. Neurosci. 2018, 2, 2514183X18793515. [CrossRef]

53. Thut, G.; Veniero, D.; Romei, V.; Miniussi, C.; Schyns, P.; Gross, J. Rhythmic TMS causes local entrainment of natural oscillatory signatures. Curr. Biol. 2011, 21, 1176-1185. [CrossRef]

54. Antal, A.; Boros, K.; Poreisz, C.; Chaieb, L.; Terney, D.; Paulus, W. Comparatively weak after-effects of transcranial alternating current stimulation (tACS) on cortical excitability in humans. Brain Stimul. 2008, 1, 97-105. [CrossRef]

55. Fröhlich, F. Endogenous and exogenous electric fields as modifiers of brain activity: Rational design of noninvasive brain stimulation with transcranial alternating current stimulation. Dialogues Clin. Neurosci. 2014, 16, 93.

56. Ahn, S.; Mellin, J.M.; Alagapan, S.; Alexander, M.L.; Gilmore, J.H.; Jarskog, L.F.; Fröhlich, F. Targeting reduced neural oscillations in patients with schizophrenia by transcranial alternating current stimulation. Neuroimage 2019, 186, 126-136. [CrossRef]

57. Alexander, M.L.; Alagapan, S.; Lugo, C.E.; Mellin, J.M.; Lustenberger, C.; Rubinow, D.R.; Fröhlich, F. Double-blind, randomized pilot clinical trial targeting alpha oscillations with transcranial alternating current stimulation (tACS) for the treatment of major depressive disorder (MDD). Transl. Psychiatry 2019, 9, 1-12. [CrossRef] [PubMed]

58. Ali, M.M.; Sellers, K.K.; Fröhlich, F. Transcranial alternating current stimulation modulates large-scale cortical network activity by network resonance. J. Neurosci. 2013, 33, 11262-11275. [CrossRef] [PubMed]

59. Force, R.B.; Riddle, J.; Jarskog, L.F.; Fröhlich, F. A case study of the feasibility of weekly tACS for the treatment of auditory hallucinations in schizophrenia. Brain Stimul. Basic Transl. Clin. Res. Neuromodul. 2021, 14, 361-363.

60. Lustenberger, C.; Boyle, M.R.; Alagapan, S.; Mellin, J.M.; Vaughn, B.V.; Fröhlich, F. Feedback-controlled transcranial alternating current stimulation reveals a functional role of sleep spindles in motor memory consolidation. Curr. Biol. 2016, 26, 2127-2136. [CrossRef] [PubMed]

61. Lam, A.D.; Noebels, J. Night watch on the titanic: Detecting early signs of epileptogenesis in Alzheimer Disease. Epilepsy Curr. 2020, 20, 369-374. [CrossRef] [PubMed]

62. Price, W.N.; Cohen, I.G. Privacy in the age of medical big data. Nat. Med. 2019, 25, 37-43. [CrossRef] [PubMed]

63. Leeming, G.; Cunningham, J.; Ainsworth, J. A Ledger of Me: Personalizing Healthcare Using Blockchain Technology. Front. Med. 2019, 6. [CrossRef]

64. Wang, T.; Lu, Y.; Cao, Z.; Shu, L.; Zheng, X.; Liu, A.; Xie, M. When Sensor-Cloud Meets Mobile Edge Computing. Sensors 2019, 19, 5324. [CrossRef] [PubMed]

65. Ren, Y.; Leng, Y.; Cheng, Y.; Wang, J. Secure data storage based on blockchain and coding in edge computing. Math. Biosci. Eng. 2019, 16, 1874-1892. [CrossRef] [PubMed]

66. Wang, J.; Warnecke, J.M.; Haghi, M.; Deserno, T.M. Unobtrusive Health Monitoring in Private Spaces: The Smart Vehicle. Sensors 2020, 20, 2442. [CrossRef] [PubMed]

67. Strain, T.; Wijndaele, K.; Dempsey, P.C.; Sharp, S.J.; Pearce, M.; Jeon, J.; Lindsay, T.; Wareham, N.; Brage, S. Wearable-devicemeasured physical activity and future health risk. Nat. Med. 2020, 26, 1385-1391. [CrossRef] [PubMed] 